\title{
Embedding and knotting of flat compact surfaces in 3-space
}

\author{
Peter Røgen
}

\begin{abstract}
In 3-space, any compact surface with nonempty boundary is isotopic to a flat (zero Gaussian curvature) surface and two such flat surfaces are isotopic through flat surfaces if and only if they are isotopic through ordinary surfaces. Hereby the isotopy classes of flat surfaces are in one-to-one correspondence with the isotopy classes of ordinary surfaces which have no constraint on their curvature. Applied to Seifert surfaces we get: Any simple closed space curve can be deformed until it bounds a flat orientable surface.
\end{abstract}

Mathematics Subject Classification (2000). 58D10, 53A05.

Keywords. Isotopy classes of flat compact surfaces in 3-space, developable surfaces, Seifert surfaces.

\section{Introduction}

A flat surface in euclidean 3-space is an embedded surface with zero Gaussian curvature everywhere. The main result of this paper, Theorem 13, is that the isotopy classes of flat surfaces are in one-to-one correspondence with the isotopy classes of ordinary surfaces which have no constraint on their curvature. Theorem 13 is the flat analogue of the main theorem in [3] by Herman Gluck and Liu-Hua Pan.

Theorem 1. (H. Gluck and L.-H. Pan, [3]) (a) In 3-space, any compact orientable surface with nonempty boundary can be deformed into one with positive curvature. (b) Any two such surfaces with positive curvature can be deformed into one another through surfaces of positive curvature if and only if they can be deformed into one another through ordinary surfaces, preserving their natural orientations.

At the end of this paper we discus the analogous problem concerning compact negatively curved surfaces with nonempty boundaries. This elaboration leads to Conjecture 14 that describes the isotopy classes of compact negatively curved surfaces with nonempty boundaries.

It is well known that a closed compact flat complete surface embedded in 3space does not exist even though the torus has a flat metric. However, part (a) of Theorem 13 ensures that a flat surface exists in every isotopy class of compact 
surfaces with nonempty boundary. Hence, the nonempty boundaries allow flat surfaces in 3-space to be arbitrarily knotted and twisted. For instance, a torus with one hole may be embedded as a flat surface in 3-space with any knot tied on it. This explains the title of this paper. Part (b) of Theorem 13 ensures that any two isotopic flat surfaces are isotopic through flat surfaces.

A simple closed curve in 3-space bounds an orientable compact embedded surface, that is, a Seifert surface. By part (a) of Theorem 13 this surface is isotopic to a flat surface. The boundary of this flat surface has the same knot type as the given curve. Hence, an immediate consequence of Theorem 13 is

Corollary 2. Any simple closed space curve can be deformed until it bounds an orientable compact embedded flat (Seifert) surface.

Corollary 2 leaves open the possibility that any simple closed space curve bounds a flat surface. In a coming paper by the author it will be shown that this is not the case and furthermore a set of necessary and in a weakened sense sufficient conditions for a knot or link to generically bound a flat immersed surface without planar regions will be given.

The strategy of the proof of Theorem 13 is: A compact connected surface with nonempty boundary deformation contracts to a "topological" spine, that is, to a finite number of simple closed curves in 3-space that all intersect in one common point. See Figure 1. Under isotopy of an embedded surface through embeddings, a topological spine is mapped to topological spines of all surfaces in the isotopy. By a small deformation it may be assumed that topological spines do not intersect the boundaries of the surfaces on which they lie.

Consider a Möbius strip and an orientable cylinder in 3-space. The topological spines of each of these two surfaces consist of one simple closed curve in 3 -space. If two such curves represent the same knot type, then the two topological spines are isotopic, but the two surfaces are not isotopic. In order to tell if two surfaces with isotopic spines are isotopic, we attach the number of times the surface "twists" $\dagger$ around each closed curve in a topological spine to this closed curve.

Definition 3. Let $\mathbf{r}$ be a simple closed curve on an embedded surface $\mathrm{S}$ in 3-space. Let $N_{\varepsilon}(\mathbf{r}) \subset \mathrm{S}$ be a tubular neighbourhood of radius $\varepsilon>0$ of $\mathbf{r}$ in $\mathrm{S}$ and let the orientations of $\mathbf{r}$ and each component of $\partial N_{\varepsilon}(\mathbf{r})$ be given by a preferred direction of traversion of $N_{\varepsilon}(\mathbf{r})$. Then the Möbius twisting number $\operatorname{Mtn}(\mathbf{r}, \mathrm{S})$, for $\varepsilon>0$ sufficiently small, is given by $\operatorname{Mtn}(\mathbf{r}, \mathrm{S})=\frac{1}{2} \operatorname{link}\left(\mathbf{r}, \partial N_{\varepsilon}(\mathbf{r})\right)$.

In the above definition, link $\left(\mathbf{r}, \partial N_{\varepsilon}(\mathbf{r})\right)$ is the total linking number between $\mathbf{r}$ and the link $\partial N_{\varepsilon}(\mathbf{r})$, that is, link $\left(\mathbf{r}, \partial N_{\varepsilon}(\mathbf{r})\right)$ is the sum of the linking numbers between $\mathbf{r}$ and all (one or two) components of $\partial N_{\varepsilon}(\mathbf{r})$. Reversing the preferred direction of traversion of $N_{\varepsilon}(\mathbf{r})$ reverses the orientation of $\mathbf{r}$ and of each component of $\partial N_{\varepsilon}(\mathbf{r})$. Hereby the linking number(s) between these curves are unchanged.

\footnotetext{
$\dagger$ The definition of this "twisting number" is given in [7], where it is denoted "the twisting number". To avoid conflict with the "twist" from "link=twist+writhe" we here introduce the phrase "Möbius twisting number" and the notation Mtn for the in [7] introduced "twisting number".
} 
The Möbius twisting number is thus independent of the direction of traversion of $N_{\varepsilon}(\mathbf{r})$ used to define it. As the linking number is invariant under ambient isotopy an immediate consequence of Definition 3 is

Proposition 4. The Möbius twisting number of a simple closed curve on an embedded surface in 3-space is invariant under isotopy through simple closed curves on the surface and invariant under isotopy of the surface through embedded surfaces in 3-space.

Consider a tubular neighbourhood of one closed curve in a spine of a surface, that is a closed strip. In [7] it is proven that a closed strip in 3-space is, up to ambient isotopy, given by the knot type and the Möbius twisting number of a simple closed curve traversing the strip once. A given surface may be reconstructed by gluing together such tubular neighbourhoods of each closed curve in a spine. It follows that if two surfaces have isotopic spines, and the Möbius twisting numbers of the corresponding closed curves in each spine are equal, then the two surfaces are in fact isotopic. Topologically, the isotopy classes of compact surfaces with nonempty boundaries may be described as follows.

Proposition 5. The isotopy class of compact surface with nonempty boundary is determined by the isotopy class of a spine of the surface with Möbius twisting numbers attached to each closed curve in the spine.

In [3] surfaces of positive curvature are considered, and the natural choice of "twisting number" is the self-linking number of each loop in the spine. The selflinking number of a given curve is the linking number between the given curve and a curve obtained by slightly pushing the given curve along the principal normals, see eg. [6]. When restricted to positive curvature surfaces, the Möbius twisting number given by Definition 3 agrees with the self-linking number. Curves on flat surfaces may have vanishing curvature, and (worse) flat surfaces may be unorientable. Hence, in the case of flat surfaces, the self-linking number can not be used as a "twisting number".

Given a curve on a surface, the envelope of the tangent planes of the surface along the curve defines (when regular) a flat surface. Neighbourhoods of this curve on the two surfaces are isotopic through the normal exponential map on either of the two surfaces. However, the above envelope fails to give a regular surfaces if the tangent of the curve is in an asymptotic direction on the surface. This is e.g. the case on the left hand side of Figure 3. For the positive curvature surfaces, considered in [3], this construction always works, but only isotopies of orientable flat surfaces may be constructed this way.

Let $S_{0}$ and $S_{1}$ be two flat surfaces isotopic through flat surfaces, let $\mathbf{a}_{0}$ be a closed curve on $\mathrm{S}_{0}$, and denote $\mathbf{a}_{0}$ 's image on $\mathrm{S}_{1}$ under this isotopy by $\mathbf{a}_{1}$. If $\mathbf{a}_{0}$ and $\mathbf{a}_{1}$ both have non-vanishing curvature, they need not have the same self-linking number. If not, then in the curve isotopy from $\mathbf{a}_{0}$ to $\mathbf{a}_{1}$, there is at least one curve with one point of zero curvature. At this point this curve is in an asymptotic direction on the corresponding surface. Even from an isotopy of flat surfaces it 


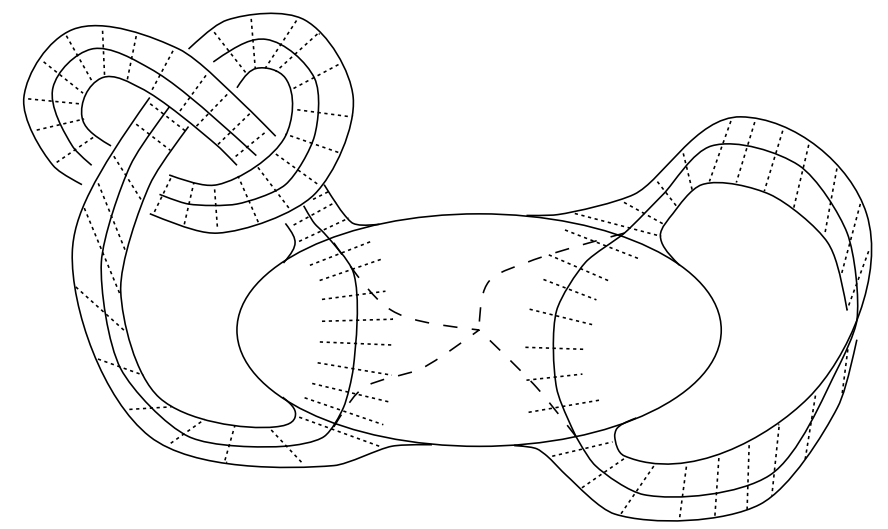

Figure 1. A flat model surface built on a slightly modified spine. The original "topological" spine is indicated by dotted arcs. The two closed strips are a plus three twisted right-hand trefoil knot and a plus one half twisted unknot (Möbius strip).

is thus in general not possible to rebuilt the surfaces as envelopes of the tangent planes along curves.

To avoid problems with the asymptotic directions, the procedure taken here is to first unwind the asymptotic directions (rulings) along the curves in the spines, as shown on figure 3, for then to construct the main part of the isotopies of flat surfaces such that the asymptotic directions (rulings) never are in the directions of the curves of the spines. Hereby a neighbourhood of each curve in a spine can be parametrized as a globally ruled flat strip.

A flat model surface is shown in Figure 1. Let $p$ be the point of intersection of the closed curves in a topological spine. Then a flat model surface built on this spine is planar in a region containing $p$ in its interior. By Proposition 4 , the Möbius twisting number is invariant under isotopy through simple closed curves on the surface. Hence, the simple closed curves of a spine may be chosen freely on the planar region of a model surface. Each closed curve in the spine is an axis of a flat globally ruled strip coinciding with the planar region, such that the closed curve has the desired Möbius twisting number with respect to this ruled strip. The planarity of the region containing $p$ makes it possible to glue the strips and this planar region together to make a regular surface.

For some closed curves the Möbius twisting numbers they can have on globally ruled flat surfaces are bounded form above, from below, or both from above and below. This is proven in the next section which also shows a way to deform a given curve isotopy such that any Möbius twisting number of flat globally ruled surfaces is obtainable.

Making the construction of flat model surfaces sufficiently canonical, an isotopy of flat model surfaces through flat model surfaces is obtained from an isotopy of a spine with Möbius twisting numbers attached. Hereby any given surface isotopy 
induces a flat model surface isotopy. The main result of this paper then follows by proving that any compact surface with nonempty boundary is isotopic to a flat model surface, and that if the given surface is flat, then there is an isotopy through flat surfaces to a flat model surface. Hence, a crucial step for the proof of Theorem 13 is to construct sufficiently canonical globally ruled flat strips. This is done in the following section.

\section{Flat closed strips in 3-space}

A closed strip in 3-space is an embedding of the Möbius strip or the orientable cylinder, both with boundary, into 3-space. In [4] it is proven that, except for the $\pm 1 / 2$-twisted unknotted (Möbius) strips, the isotopy class of a closed strip is uniquely given by the knot type of its boundary or by the oriented link type of its boundary, in case of orientable closed strips.

Proposition 6. Let $\mathbf{r}$ be a simple closed space curve. Assume the curvature of $\mathbf{r}$ vanishes only on a finite set of intervals and points and assume the limit of the torsion of $\mathbf{r}$ vanishes wherever curvature vanishes. Then the following two statements are equivalent.

For any half integer $t$, the curve $\mathbf{r}$ is an axis of a flat ruled surface $\mathrm{S}_{t}$, such that, $\operatorname{Mtn}\left(\mathbf{r}, \mathrm{S}_{t}\right)=t$.

The torsion of $\mathbf{r}$ takes both signs.

Furthermore, we may choose two intervals $\mathrm{I}_{+}$and $\mathrm{I}_{-}$with positive resp. negative torsion on which the rulings are steered and on the remainder of $\mathbf{r}$ the rulings may be chosen orthogonal to $\mathbf{r}$.

Remark 7. From the proof of Proposition 6 below it follows that on an interval with orthogonal rulings, these rulings are uniquely given when one ruling is specified in one point. Hereby, Proposition 6 gives a, for our purpose, sufficiently canonical construction of flat globally ruled closed strips. For related results see [1].

Before proceeding with the proof of Proposition 6, we need to introduce some notation and to do calculations leading to Equation 2.5 that steers the possible choices of vector fields giving flat ruled surfaces along a given axis. Let $\mathbf{r}: \mathbb{R} / \mathrm{L} \mathbb{Z} \rightarrow$ $\mathbb{R}^{3}$ be a closed space curve parametrized by arc length $s$, and let $\mathbf{v}$ be a choice of trivialization of the normal bundle, i.e., a closed unit normal vector field $\mathbf{v}$, along $\mathbf{r}$, such that $\operatorname{link}(\mathbf{r}, \mathbf{r}+\varepsilon \mathbf{v})=0$ for $\varepsilon>0$ sufficiently small. If $\mathbf{t}$ denotes the unit tangent vectors to $\mathbf{r}$ and $\mathbf{u}=\mathbf{t} \times \mathbf{v}$, then $\{\mathbf{t}, \mathbf{v}, \mathbf{u}\}$ is an orthonormal basis for $\mathbb{R}^{3}$ for each point on $\mathbf{r}$. By orthogonality, there are Frenet like equations

$$
\begin{array}{rlrrr}
\mathbf{t}^{\prime} & = & a \mathbf{v} & +b \mathbf{u} \\
\mathbf{v}^{\prime} & = & -a \mathbf{t} & & +c \mathbf{u} \\
\mathbf{u}^{\prime} & = & -b \mathbf{t} & -c \mathbf{v} &
\end{array}
$$

where primes indicate differentiation with respect to $s$ and $a, b, c: \mathbb{R} / \mathrm{L} \mathbb{Z} \rightarrow \mathbb{R}$ are periodic functions. 
A ruled surface with $\mathbf{r}$ as axis is given by $\mathbf{f}(s, t)=\mathbf{r}(s)+t \mathbf{q}(s)$ for some vector field $\mathbf{q}$ along $\mathbf{r}$. This surface is regular if

$$
\mathbf{0} \neq \frac{\partial \mathbf{f}(s, t)}{\partial s} \times \frac{\partial \mathbf{f}(s, t)}{\partial t}=\left(\mathbf{t}(s)+t \mathbf{q}^{\prime}(s)\right) \times \mathbf{q}(s) .
$$

Along $\mathbf{r}$, that is for $t=0$, this gives $\frac{\partial \mathbf{f}(s, 0)}{\partial s} \times \frac{\partial \mathbf{f}(s, 0)}{\partial t}=\mathbf{t}(s) \times \mathbf{q}(s)$. By continuity and compactness the ruled surface is regular in a neighbourhood of $\mathbf{r}$ if $\mathbf{q}$ is never parallel to the tangents to $\mathbf{r}$. Hereby $\mathbf{q}$ may be written as

$$
\mathbf{q}=\alpha \mathbf{t}+\cos \theta \mathbf{v}+\sin \theta \mathbf{u}
$$

where $\alpha, \theta: \mathbb{R} / \mathrm{L} \mathbb{Z} \rightarrow \mathbb{R}$ are cylinder coordinates, see Figure 2. Using these coordinates, the ruled surface closes up if and only if there exists an integer $p$ such that $\theta(\mathrm{L})-\theta(0)=\pi p$ and $\alpha(\mathrm{L})=(-1)^{p} \alpha(0)$. As $\operatorname{link}(\mathbf{r}, \mathbf{r}+\varepsilon \mathbf{v})=0$ for $\varepsilon>0$ sufficiently small, the axis $\mathbf{r}$ has Möbius twisting number $\operatorname{Mtn}(\mathbf{r}, \mathbf{f})=p / 2$ on such a ruled surface.

As $\frac{\partial^{2} \mathbf{f}}{\partial t^{2}}=0$ the Gaussian curvature $\mathrm{K}=\frac{-m^{2}}{\left|\frac{\partial \mathbf{f}}{\partial s} \times \frac{\partial \mathbf{f}}{\partial t}\right|^{2}}=\frac{-\left[\frac{\partial^{2} \mathbf{f}}{\partial s \partial t} \frac{\partial \mathbf{f}}{\partial s} \frac{\partial \mathbf{f}}{\partial t}\right]}{\left|\frac{\partial \mathbf{f}}{\partial s} \times \frac{\partial \mathbf{f}}{\partial t}\right|^{2}}$ is zero if

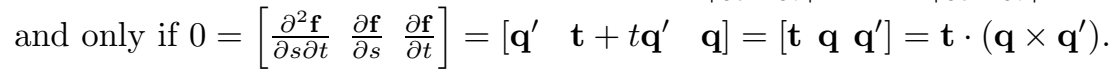

$$
\begin{aligned}
\mathbf{q}^{\prime}= & \alpha^{\prime} \mathbf{t}+\alpha a \mathbf{v}+\alpha b \mathbf{u}-\theta^{\prime} \sin \theta \mathbf{v}-a \cos \theta \mathbf{t}+c \cos \theta \mathbf{u} \\
& +\theta^{\prime} \cos \theta \mathbf{u}-b \sin \theta \mathbf{t}-c \sin \theta \mathbf{v} \\
0= & {\left[\begin{array}{lll}
\mathbf{t} & \mathbf{q} & \mathbf{q}^{\prime}
\end{array}\right] } \\
= & \left|\begin{array}{ccc}
1 & \star & \star \\
0 & \cos \theta & \alpha a-\theta^{\prime} \sin \theta-c \sin \theta \\
0 & \sin \theta & \alpha b+\theta^{\prime} \cos \theta+c \cos \theta
\end{array}\right| \\
= & \alpha b \cos \theta+\theta^{\prime}+c-\alpha a \sin \theta \\
& \mathbb{} \\
\theta^{\prime}= & \alpha(a \sin \theta-b \cos \theta)-c
\end{aligned}
$$

We can rewrite $\mathbf{t}^{\prime}$ setting $a=\kappa \cos \phi$ and $b=\kappa \sin \phi$. See Figure 2. For $\kappa>0$, the angle $\phi$ is well-defined up to an integral multiple of $2 \pi$. This gives

$$
\theta^{\prime}=\alpha \kappa(\sin \theta \cos \phi-\cos \theta \sin \phi)-c=\alpha \kappa \sin (\theta-\phi)-c .
$$

The famous formula: link equals twist plus writhe holds if curvature has zeros [5]. The linking number between $\mathbf{r}$ and $\mathbf{r}+\varepsilon \mathbf{v}$ is chosen to be zero. So it follows that

$$
\mathrm{T} w(\mathbf{r}, \mathbf{v})=\frac{1}{2 \pi} \int_{0}^{\mathrm{L}} \mathbf{v}(s)^{\prime} \cdot \mathbf{u}(s) d s=\frac{1}{2 \pi} \int_{0}^{\mathrm{L}} c(s) d s=-\mathrm{Wr}(\mathbf{r}) .
$$

For $\alpha \equiv 0$ and $\theta$ constant, Equation (2.1) yields that the ruled surface given by $\mathbf{r}(s)+t(\cos (\theta) \mathbf{v}(s)+\sin (\theta) \mathbf{u}(s))$ is flat if and only if $c \equiv 0$. Let $\tilde{\mathbf{v}}$ be the unique unit normal vector field along $\mathbf{r}$ such that $\tilde{\mathbf{v}}(0)=\mathbf{v}(0)$ and $\tilde{\mathbf{v}}$ has $c \equiv 0$. Note, that in 


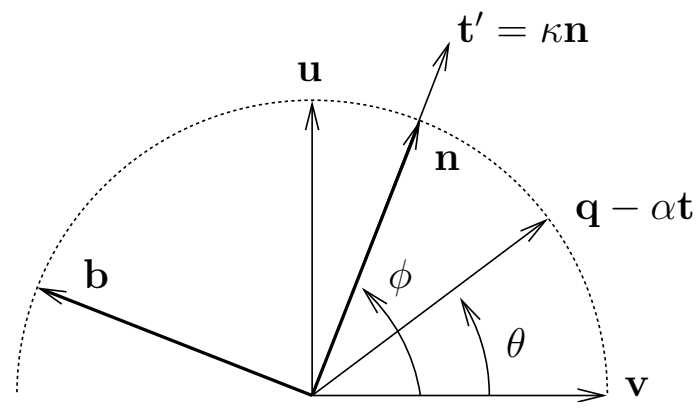

Figure 2. The Frenet frame and the projection $\mathbf{q}-\alpha \mathbf{t}$ of the ruling $\mathbf{q}$ in the normal plane spanned by the frame $\mathbf{v}, \mathbf{u}$.

general $\tilde{\mathbf{v}}$ does not close up since $\tilde{\mathbf{v}}$ is twisted $\mathrm{T} w(\mathbf{r}, \mathbf{v})$ less than $\mathbf{v}$. Using this new (non-closed) frame $\tilde{\mathbf{v}}$ and setting $\tilde{\mathbf{u}}=\mathbf{t} \times \tilde{\mathbf{v}}$, we obtain $\mathbf{q}=\alpha \mathbf{t}+\cos \theta \mathbf{v}+\sin \theta \mathbf{u}=$ $\alpha \mathbf{t}+\cos \tilde{\theta} \tilde{\mathbf{v}}+\sin \tilde{\theta} \tilde{\mathbf{u}}$ and

$$
\tilde{\theta}^{\prime}=\alpha \kappa \sin (\tilde{\theta}-\tilde{\phi}) .
$$

A necessary and sufficient condition for the flat surface to close up and to have Möbius twisting number $\operatorname{Mtn}(\mathbf{r}, \mathbf{f})=p / 2$ for a given $p \in \mathbb{Z}$ is that

$$
\tilde{\theta}(\mathrm{L})-\tilde{\theta}(0)=p \pi-\mathrm{Wr}(\mathbf{r}) \quad \text { and } \quad \alpha(\mathrm{L})=(-1)^{p} \alpha(0) .
$$

We need to describe the frame $\tilde{\mathbf{v}}$ using the Frenet Apparatus. For this let I be an interval where $\mathbf{r}$ has positive curvature. By orthogonality, $\tilde{\mathbf{v}}$ may be written as $\tilde{\mathbf{v}}=\cos v \mathbf{n}+\sin v \mathbf{b}$ and the Frenet equations give

$$
\tilde{\mathbf{v}}^{\prime}=-v^{\prime} \sin v \mathbf{n}+\cos v(-\kappa \mathbf{t}+\tau \mathbf{b})+v^{\prime} \cos v \mathbf{b}-\tau \sin v \mathbf{n} .
$$

The ruled surface with $\mathbf{r}$ as axis and $\tilde{\mathbf{v}}$ as rulings is flat if and only if

$$
\begin{aligned}
0 & =c=\left[\begin{array}{lll}
\mathbf{t} & \tilde{\mathbf{v}} & \tilde{\mathbf{v}}^{\prime}
\end{array}\right] \\
& =\left|\begin{array}{ccc}
1 & \star & \star \\
0 & \cos v & -v^{\prime} \sin v-\tau \sin v \\
0 & \sin v & \tau \cos v+v^{\prime} \cos v
\end{array}\right| \\
& =\tau+v^{\prime}
\end{aligned}
$$

Hence, on the interval I, where $\mathbf{r}$ has positive curvature, the vector field $\tilde{\mathbf{v}}$ is given by

$$
\tilde{\mathbf{v}}(s)=\cos \left(-\int_{s_{0}}^{s} \tau(s) d s+k_{\mathrm{I}}\right) \mathbf{n}(s)+\sin \left(-\int_{s_{0}}^{s} \tau(s) d s+k_{\mathrm{I}}\right) \mathbf{b}(s)
$$

for some constant $k_{\mathrm{I}}$. On a straight segment of $\mathbf{r}$ (where $\mathbf{r}$ has zero curvature), a similar calculation shows that the vector field $\tilde{\mathbf{v}}$ is constant on this segment. By the assumption that torsion vanishes whenever curvature vanishes, the vector field 
$\tilde{\mathbf{v}}$ is $\mathrm{C}^{1}$ along $\mathbf{r}$. From the equations

$$
\begin{aligned}
\mathbf{t}^{\prime}= & \kappa \mathbf{n} \\
= & \kappa \cos \tilde{\phi} \tilde{\mathbf{v}}+\kappa \sin \tilde{\phi} \tilde{\mathbf{u}} \\
= & \kappa \cos \tilde{\phi}\left(\cos \left(-\int \tau(s) d s+k_{\mathrm{I}}\right) \mathbf{n}+\sin \left(-\int \tau(s) d s+k_{\mathrm{I}}\right) \mathbf{b}\right) \\
& +\kappa \sin \tilde{\phi}\left(-\sin \left(-\int \tau(s) d s+k_{\mathrm{I}}\right) \mathbf{n}+\cos \left(-\int \tau(s) d s+k_{\mathrm{I}}\right) \mathbf{b}\right)
\end{aligned}
$$

it follows that

$$
\begin{aligned}
1 & =\cos \tilde{\phi} \cos \left(-\int_{s_{0}}^{s} \tau(s) d s+k_{\mathrm{I}}\right)-\sin \tilde{\phi} \sin \left(-\int_{s_{0}}^{s} \tau(s) d s+k_{\mathrm{I}}\right) \\
& =\cos \left(\tilde{\phi}-\int_{s_{0}}^{s} \tau(s) d s+k_{\mathrm{I}}\right) .
\end{aligned}
$$

Hence,

$$
\tilde{\phi}(s)=\int_{s_{0}}^{s} \tau(s) d s-k_{\mathrm{I}} \text { modulo } 2 \pi .
$$

Thus if $\kappa>0$ on I, then for $s \in \mathrm{I}$, Equation (2.2) may be written as

$$
\tilde{\theta}^{\prime}(s)=\alpha(s) \kappa(s) \sin \left(\tilde{\theta}(s)-\int_{s_{0}}^{s} \tau(s) d s+k_{\mathrm{I}}\right) .
$$

If $\kappa=0$ then $\tilde{\theta}^{\prime}=0$ and $\tilde{\mathbf{v}}^{\prime}=\tilde{\mathbf{u}}^{\prime}=\mathbf{0}$.

Proof of Proposition 6. The strategy for constructing flat ruled strips in the following is to freely choose $\alpha$ whereby $\tilde{\theta}$ is given by Equation 2.5 and an initial value of $\tilde{\theta}$. If $\tau>0$ on some interval $\mathrm{I}_{+}$, then, by a proper choice of $\alpha, \tilde{\theta}$ can decrease arbitrarily much on this interval and if $\tau<0$ on an interval I- then $\tilde{\theta}$ can increase arbitrarily much on this interval. Assuming the torsion takes both signs, such intervals, $\mathrm{I}_{+}$and $\mathrm{I}_{-}$, may be chosen not to contain 0 . Choosing $\alpha=0$ on $[0, \mathrm{~L}] \backslash\left(\mathrm{I}_{+} \cup \mathrm{I}_{-}\right)$the angle $\tilde{\theta}$ is constant on $[\underset{\sigma}{0}, \mathrm{~L}] \backslash\left(\mathrm{I}_{+} \cup \mathrm{I}_{-}\right)$. By controlling $\alpha$ on $\mathrm{I}_{+}$and $\mathrm{I}_{-}$, the desired difference, see $(2.3), \tilde{\theta}(\mathrm{L})-\tilde{\theta}(0)=p \pi-\mathrm{Wr}(\mathbf{r})$ can be obtained for any $p \in \mathbb{Z}$ and $\alpha(\mathrm{L})=(-1)^{p} \alpha(0)=0$ is trivially fulfilled. On the other hand, if the torsion of $\mathbf{r}$ has constant sign, then the possible Möbius twisting numbers are, as a consequence of Equation (2.5), bounded either from above or from below.

Consider an isotopy between two closed curves such that all curves in this isotopy possess points with both positive and negative torsion. Proposition 6 ensures that for any given Möbius twisting number each curve in this isotopy is an axis of a globally ruled closed strip giving the desired Möbius twisting number. This will be made into an isotopy of flat closed strips below. This construction is the cornerstone in the proof of the following lemma. As mentioned in the 



Figure 3. Left, an unavoidable non transversal intersection between curve and rulings. Right, a local deformation of the surface (pulling the label off the bottle), such that the deformed surface can be locally parametrized as a ruled surface.

introduction, this lemma is crucial for the proof of Theorem 13, and it was posed as a question to the author by Herman Gluck.

Lemma 8. Two flat closed strips in 3-space are isotopic through ordinary closed strips in 3-space if and only if they are isotopic through flat closed strips.

Proof. Let $H_{u}, u \in[0,1]$, be an isotopy between two flat closed strips $H_{0}$ and $H_{1}$ and let $\mathbf{a}_{i} \subset H_{i}$ be a topological spine of $H_{i}, i=0,1$. Closed strips may, by contracting the boundaries, be considered as neighbourhoods of their spines.

Sublemma 9. A neighbourhood of $\mathbf{a}_{i}$ on $H_{i}$ can (possibly modulo an isotopy of $H_{i}$ ) be parametrized as a globally ruled flat surface with $\mathbf{a}_{i}$ as axis. I.e., $\mathbf{a}_{i}$ is transversal to the rulings of $H_{i}$.

Proof. By the characterization of flat surfaces given in [8], the surface $H_{i}$ is piecewise ruled. That is, on a compact subset of $H_{i}$, namely the closure of the set of points, called parabolic points, where one of the principal curvatures is non-zero, rulings are given by the zero principal curvature directions. We call these the ruled regions. The remainder of $H_{i}$ consists of planar points, i.e., points with both principal curvatures equal to zero, and is indeed a union of planar regions. Within a neighbourhood of a curve a planar region can be parametrized as a ruled surface with this curve as axis. The only restriction is that the rulings must be chosen in the plane defined by the region and the rulings may not cross the tangents to the curve in order to get a regular surface containing the curve.

The ruled regions of $H_{i}$ form a compact set. Hence, the spine $\mathbf{a}_{i}$ may be chosen such that $\mathbf{a}_{i}$ has transversal intersections with the rulings except in a finite number of points. Figure 3 shows why non-transversal intersections are in general unavoidable and it indicates how this problem may be avoided by a slight isotopy that makes the surface planar in a neighbourhood of a non-transversal intersection. 
Let $q$ be a point of non transversal intersection between a ruling and a spine. The parabolic points are dense in the ruled regions of $H_{i}$. Hence, perhaps by a slight deformation of $\mathbf{a}_{i}$, the point $\mathbf{q}$ may be assumed to be a parabolic point on $H_{i}$.

Consider the unique curve $\mathbf{c}$ on $H_{i}$ through $\mathbf{q}=\mathbf{c}(0)$ and orthogonal to the rulings. Let $\mathbf{v}$ be a vector field along $\mathbf{c}$ giving the directions of the rulings of the surface. In a neighbourhood of $\mathbf{q}$, the surface is given by $\mathbf{f}(s, t)=\mathbf{c}(s)+t \mathbf{v}(s)$, $-\varepsilon \leq s \leq \varepsilon$. The curve $\mathbf{a}_{i}$ is locally given by $\mathbf{f}(g(t), t)$, where $g(0)=0, g^{\prime}(0)=0$, and it may be assumed that $g^{\prime \prime}(0)<0$. As $\mathbf{q}$ is a parabolic point, one of the principal curvatures $k \neq 0$. By the choice of the curve $\mathbf{c}$, this curve has curvature greater than or equal to $|k|$ at the point $\mathbf{q}$. Consider the plane $\mathrm{P}$ through $\mathbf{q}$ orthogonal to the ruling through $\mathbf{q}$ and the projection of $\mathbf{c}$ onto $\mathrm{P}, \pi(\mathbf{c})$. An isotopy of a neighbourhood of $\mathbf{q}$ on $\mathbf{c}$ to $\pi(\mathbf{c})$ along normals to $\mathrm{P}$ can be constructed using a partition of unity. As $\pi(\mathbf{c})$ has curvature $|k|>0$ at $\mathbf{q}$, non-vanishing curvature is preserved during this isotopy. Hereby the torsion vanishes on this neighbourhood. Within the plane $\mathrm{P}$ the projection $\pi(\mathbf{c})$ is made straight in a smaller neighbourhood of $\mathbf{q}$.

An isotopy, as constructed in the proof of Proposition 6, of $H_{i}$ that makes $H_{i}$ planar in a region containing $\mathbf{q}$ in its interior can be constructed using the rulings given by $\mathbf{v}_{u}(-\varepsilon)=\mathbf{v}(-\varepsilon), \mathbf{v}_{u}$ is orthogonal to $\mathbf{c}_{u}$, and the ruled surface given by $\mathbf{f}_{u}(s, t)=\mathbf{c}_{u}(s)+t \mathbf{v}_{u}(s)$ is flat. By compactness of $H_{i}$ this isotopy can be assumed to go through embeddings.

On the deformed part of the surface, now given by $\mathbf{f}_{1}(s, t)=\mathbf{c}_{1}(s)+t \mathbf{v}_{1}(s)$, the curve given by $t \mapsto \mathbf{f}_{1}(g(t), t)$ lies in a plane for $t$ in a neighbourhood of zero. Note, that since the parametrization of this curve is fixed with respect to axes and rulings, all transversal intersections have remained so during the constructed isotopy. In the constructed planar region, rulings may be chosen such that they are all transversal to the curve. See Figure 3. A neighbourhood of the curve given by $t \mapsto \mathbf{f}_{1}(g(t), t)$ on the isotoped surface may thus be parametrized as a ruled surface with this curve as axis.

By such local isotopies that only concern neighbourhoods of the non transversal intersections between $\mathbf{a}_{i}$ and the rulings of $H_{i}$, a curve $\tilde{\mathbf{a}}_{i}$ on $\widetilde{H}_{i}$ is obtained, such that a neighbourhood of $\tilde{\mathbf{a}}_{i}$ on $\widetilde{H}_{i}$ can be parametrized as a (globally) ruled surface with $\tilde{\mathbf{a}}_{i}$ as axis. Note that on a flat globally ruled Möbius strip the rulings globally are only projectively well-defined.

Sublemma 10. The axis $\mathbf{a}_{i}$ of $H_{i}$ can (possibly modulo an isotopy of $H_{i}$ ) be assumed to have non-vanishing curvature.

Proof. Recall that the curvature of $\mathbf{a}_{i}$ as space curve $\kappa$, the geodesic curvature $\kappa_{g}$, and the normal curvature $\kappa_{n}$, fulfill the equation $\kappa^{2}=\kappa_{g}^{2}+\kappa_{n}^{2}$. By Sublemma 9, $\mathbf{a}_{i}$ is transversal to the rulings of $H_{i}$. So the normal curvature $\kappa_{n}(s)$ is zero if and only if $\mathbf{a}_{i}(s)$ is a planar point. Hence, the curvature of $\mathbf{a}_{i}$ vanishes if and only if $\mathbf{a}_{i}$ has zero geodesic curvature in a planar point of $H_{i}$. 


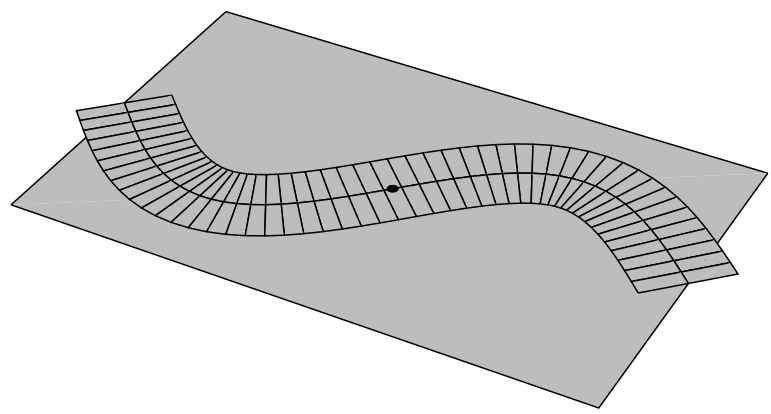

Figure 4. An unavoidable vanishing of curvature of spines on a planar region of a flat surface.

First, consider the case that $\mathbf{a}_{i}$ has zero curvature in $\mathbf{a}_{i}(s)$ and that $\mathbf{a}_{i}(s)$ does not lie in the interior of a planar region of $H_{i}$. By a local isotopy of $\mathbf{a}_{i}$ on $H_{i}$ through axes of $H_{i}$, the zero of $\mathbf{a}_{i}$ 's geodesic curvature can be moved to a parabolic point giving $\mathbf{a}_{i}$ non-vanishing curvature at $\mathbf{a}_{i}(s)$.

Otherwise, $\mathbf{a}_{i}$ has zero curvature in $\mathbf{a}_{i}(s)$ and a neighbourhood of $\mathbf{a}_{i}(s)$ on $H_{i}$ consists of planar points only. See Figure 4. Let $\mathrm{R}$ denote the planar region of $H_{i}$ containing $\mathbf{a}_{i}(s)$, and let $\mathrm{P}$ be the plane containing $\mathrm{R}$. Deforming $\mathbf{a}_{i}$ on $\mathrm{R}$, it may be assumed that the geodesic curvature of $\mathbf{a}_{i}$ vanishes in a finite number points only. Let $\mathbf{a}_{i}(s)$ denote one of these points. Deforming along the normals to $\mathrm{P}$, and thus fixing the projection of $\mathbf{a}_{i}$ onto the plane $\mathrm{P}$, a neighbourhood of $\mathbf{a}_{i}(s)$ on $H_{i}$ may be lifted through cylinder surfaces to make the normal curvature of the deformed $\mathbf{a}_{i}$ at $\mathbf{a}_{i}(s)$ non-zero. As the projection of the deformed surface into the plane $\mathrm{P}$ only has vanishing curvature at the point $\mathbf{a}_{i}(s)$, this finally gives $\mathbf{a}_{i}$ non-vanishing curvature everywhere.

Sublemma 11. The self-linking numbers of the axes $\mathbf{a}_{0}$ and $\mathbf{a}_{1}$ can (possibly modulo an isotopy of $H_{i}$ ) be assumed to be equal.

Proof. Using an isotopy as constructed in Sublemma 9, it may be assumed that $H_{0}$ has a planar region $\mathrm{R}$ and by Sublemma 10 it may be assumed that the $\mathbf{a}_{i}$, $i=0,1$, have non-vanishing curvature. Hereby the self-linking numbers of $\mathbf{a}_{0}$ and $\mathbf{a}_{1}$ are defined, but they need not be equal.

Figure 5 shows how to increase the self-linking number by one using an isotopy through flat surfaces. By interchanging up and down on this figure the self-linking number is instead decreased by one. Hence, by inserting a finite number of "bumps" on the planar region R, any given self-linking number can be obtained.

Sublemma 12. The torsion of the axis $\mathbf{a}_{i}$ can (possibly modulo an isotopy of $H_{i}$ ) be assumed to take both signs (preserving non-vanishing curvature and the 

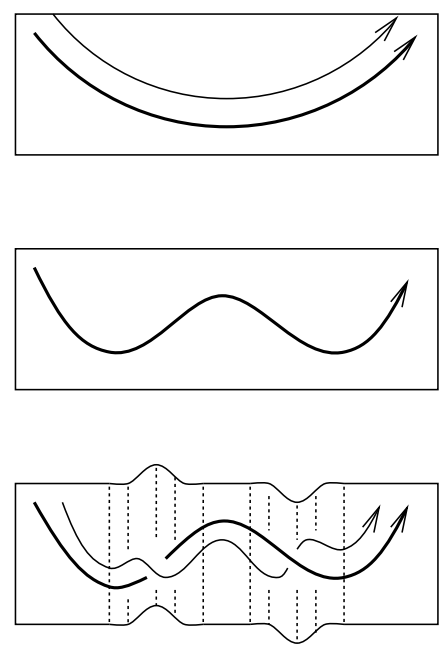

Figure 5. On the top a planar space curve (the thick curve) together with a curve pushed off along its principal normals. At the bottom a flat surface obtained by an obvious isotopy of the above plane through flat surfaces. The left bump goes away from the viewer and the right bump goes towards the viewer. The (thick) curve on the flat surface has positive curvature and the (thin) curve is pushed off along its principal normals. The two crossings between these curves are positive. Hence, the self-linking number of the thick curve is increased by one.

\section{self-linking number of $\mathbf{a}_{i}$ ).}

Proof. By an isotopy, eg. as indicated on Figure 5, it may be assumed that $H_{i}$ does not lie in a plane. Hence, there is a region of $H_{i}$ in which one of the principal curvatures $k$ is non-zero. By Proposition 2 in [8] p. 279, the geodesic torsion, i.e. the torsion of a geodesic curve with unit tangent $\mathbf{x}$ (see Prop 3 in [8] p. 281), is $\tau_{g}(\mathbf{x})=k \sin \theta \cos \theta$, where $\theta$ is the angle between $\mathbf{x}$ and the principal direction with zero principal curvature. As the principal curvature $k \neq 0$ in the considered region, an axis can be isotoped to contain a geodesic segment with positive torsion and a geodesic segment with negative torsion.

By sublemmas 10 and 11, there exists an isotopy between the two axes through positive curvature curves. This fact is due to H. Gluck and L.-H. Pan [3] and is more detailed described in [2]. Denote such an positive curvature isotopy between the axes $\mathbf{a}_{i}$ of $H_{i}, i=0,1$, by $\mathbf{a}_{u}(s)=\mathbf{a}(s, u): \mathbb{S}^{1} \times[0,1] \rightarrow \mathbb{R}^{3}$. This curve isotopy may be assumed to be smooth as it is made by performing a finite number of smooth versions of Reidemeister moves. In particular, the non-vanishing curvature and the torsion vary continuously with the family parameter $u$.

On each curve $\mathbf{a}_{u}$, consider the osculating plane $\mathrm{P}_{u}$ to the point $\mathbf{a}_{u}(0)$. By compactness, there is a common $\varepsilon$-ball around $\mathbf{a}_{u}(0)$ in $\mathrm{P}_{u}$ such that the planar projection of $\mathbf{a}_{u}$ to $\mathrm{P}_{u}$ is regular and has non-vanishing curvature for all $u \in[0,1]$. 
Fixing these planar projections, all curves can be isotoped to be planar within an $\epsilon / 2$-ball of $\mathbf{a}_{u}(0)$ preserving non-vanishing curvature.

For $\varepsilon / 2>\delta>0$, there is a family $\mathrm{A}_{u}, u \in[0,1]$, of affine transformations of 3 space, such that, $\mathrm{A}_{u}\left(\mathbf{a}_{u}(-\delta)\right)=(0,0,0)$ and $\mathrm{A}_{u}\left(\mathbf{a}_{u}(\delta)\right)=(1,0,0)$ for all $u \in[0,1]$ and such that $\mathrm{A}_{u}\left(\mathrm{P}_{u}\right)$ is the $x y$-plane. Note, that the image of a flat surface under an affine transformation is flat. Furthermore, affine transformations map rulings to rulings. Choosing $\delta>0$ sufficiently small, compactness ensures that the $x$-axis can be used as a parameter of the pieces of the curves $s \mapsto \mathrm{A}_{u}\left(\mathbf{a}_{u}(s)\right)$ lying in between $(0,0,0)$ and $(1,0,0)$. Hence, locally the curve isotopy is given by $[0,1] \times[0,1] \ni(x, u) \mapsto\left(x, f_{u}(x), 0\right)$. Now all $f_{u}$ are isotoped to be identical for $x \in[1 / 3,2 / 3]$ and to give positive curvature on a slightly larger interval. Points with zero curvature may be introduced, but as they occur in a plane they do not disturb the construction of rulings that still remains. See Proposition 6. The constructed planar curve-piece that is identical for all curves in the isotopy is now rolled onto a cylinder (as on Figure 3 read from the right hand side to the left hand side). By the proof of Sublemma 12, the resulting space curve-piece has both positive and negative torsion which may even be chosen constant on two sub-intervals using circular helices. This common curve segment is referred to as the $\pm \tau$-segment. We are now in possession of the requisite axes. Hence, we need only specify their rulings to complete the proof.

Along the curve $s \mapsto \mathrm{A}_{0}\left(\mathbf{a}_{0}(s)\right.$ ) (or just $\mathrm{A}_{0}\left(\mathbf{a}_{0}\right)$ ), there is a ruling vector field $\mathbf{q}_{0}$ parametrizing a neighbourhood of $\mathrm{A}_{0}\left(\mathbf{a}_{0}\right)$ on $\mathrm{A}_{0}\left(H_{0}\right)$. This vector field is given by the image under $\mathrm{A}_{0}$ of the rulings of $H_{0}$ along $\mathbf{a}_{0}$. Specifying one ruling $\mathbf{q}_{0}\left(s^{\star}\right)$, the rulings along $\mathrm{A}_{0}\left(\mathbf{a}_{0}\right)$ may be changed continuously to be orthogonal to the curve and equal to $\mathbf{q}_{0}\left(s^{\star}\right)$ at $\mathrm{A}_{0}\left(\mathbf{a}_{0}\left(s^{\star}\right)\right)$ except for one segment where $\mathrm{A}_{0}\left(\mathbf{a}_{0}\right)$ has positive torsion and another with negative torsion. The existence of these segments is ensured by Sublemma 12. The twisting of the rulings that occur during this change is compensated for within these two segments. Also the twisting of the rulings, caused by keeping the rulings orthogonal to the isotoped segment under the isotopy inserting the $\pm \tau$-segment, is compensated for within these two segments. Fixing the space curve and one ruling outside the $\pm \tau$-segment, the rulings may be isotoped to be orthogonal to the curve outside the $\pm \tau$-segment while only compensating inside the $\pm \tau$-segment.

During the curve isotopy from $\mathrm{A}_{0}\left(\mathbf{a}_{0}\right)$ to $\mathrm{A}_{1}\left(\mathbf{a}_{1}\right)$ (with the $\pm \tau$-segment inserted on all curves) the rulings outside the $\pm \tau$-segment are given by a choice of one orthogonal ruling in one point of each curve and demanding that the rulings are orthogonal to the curves and that they give a flat surface. Hereby the rulings at the endpoints of the $\pm \tau$-segment vary continuously during the isotopy. This makes it possible to control the rulings on the $\pm \tau$-segment to match the boundary conditions of Equation (2.5).

By performing the preparations of the rulings on $\mathrm{A}_{0}\left(\mathbf{a}_{0}\right)$ and the curve itself "time reversed" on $\mathrm{A}_{1}\left(\mathbf{a}_{1}\right)$ the curve isotopy from $\mathrm{A}_{0}\left(\mathbf{a}_{0}\right)$ to $\mathrm{A}_{1}\left(\mathbf{a}_{1}\right)$ gives rise to a surface isotopy through globally ruled flat strips from $\mathrm{A}_{0}\left(H_{0}\right)$ to $\mathrm{A}_{1}\left(H_{1}\right)$. Pulling 
this isotopy back using the affine mappings $\mathrm{A}_{u}$, the desired isotopy from $H_{0}$ to $H_{1}$ through globally ruled flat strips is constructed.

\section{Flat surfaces in 3 -space}

We are now ready to prove the main theorem of this paper, which implies that the isotopy classes of flat surfaces are in one-to-one correspondence with the isotopy classes of ordinary surfaces which have no curvature constraint.

Theorem 13. (a) In 3-space, any compact surface with nonempty boundary is isotopic to a flat surface. (b) Two such flat surfaces are isotopic through flat surfaces if and only if they are isotopic through ordinary surfaces.

Proof. Part (b). Let $\mathrm{S}_{u}, u \in[0,1]$, be an isotopy between two flat compact connecteded surfaces with nonempty boundaries. In order to prove part (b), an isotopy from $\mathrm{S}_{0}$ to $\mathrm{S}_{1}$ through flat surfaces must be constructed.

Let $s_{0} \subset \mathrm{S}_{0}$ be a topological spine of $\mathrm{S}_{0}$ and denote the images of this spine under the isotopy by $s_{u} \subset \mathrm{S}_{u}$. Similarly let $\mathbf{p}_{0} \in s_{0}$ be the intersection point of the closed curves in $s_{0}$, and let $\mathbf{p}_{u} \in \mathrm{S}_{u}$ be $\mathbf{p}_{0}$ 's images under the isotopy. From now on only neighbourhoods of the spines $s_{u}$ on the surfaces $\mathrm{S}_{u}$ are considered.

By local isotopies, as constructed in the proof of Sublemma 9, it may be assumed that $S_{0}$ is planar in a neighbourhood of $\mathbf{p}_{0}$ and that $S_{1}$ is planar in a neighbourhood of $\mathbf{p}_{1}$. A partition of unity between $S_{u}$ and the tangent plane to $\mathrm{S}_{u}$ at $\mathbf{p}_{u}$ makes $\mathrm{S}_{u}$ planar in an $\varepsilon$-neighbourhood of $\mathbf{p}_{u}$. By compactness, there is a common $\varepsilon>0$ such that all the surfaces, $\mathrm{S}_{u}$, can be locally isotoped through embeddings to be planar in an $\varepsilon$-neighbourhood of the images of $\mathbf{p}_{0}$ on each surface. Thus it may be assumed that each surface $\mathrm{S}_{u}$ is planar in an $\varepsilon$-neighbourhood of $\mathbf{p}_{u}$ for a fixed $\varepsilon>0$.

By Proposition 4 a simple closed curve a on $\mathrm{S}_{0}$ has a Möbius twisting number $\operatorname{Mtn}\left(\mathbf{a}, \mathrm{S}_{0}\right)$ that is invariant under isotopy of $\mathbf{a}$ on $\mathrm{S}_{0}$. So the closed curves in the spine $s_{0}$ may be chosen freely on the planar $\varepsilon$-neighbourhood of $\mathbf{p}_{0}$ of $\mathrm{S}_{0}$. Hence, the spine $s_{0}$, and thus also its images $s_{u}$, each may considered as a planar $\varepsilon$-disk together with a finite number of simple curves $\mathbf{a}_{u}^{i}$ starting and ending in pairwise disjoint points on the boundary of this disk. Furthermore, each of these curves has a Möbius twisting number attached.

The idea of this proof is as follows: A surface isotopy is given. This isotopy induces curve isotopies $\mathbf{a}_{u}^{i}, u \in[0,1]$ of the curves in the spine. Then an isotopy of closed flat strips $\mathrm{S}_{u}^{i}, u \in[0,1]$, is constructed such that the $\mathbf{a}_{u}^{i}$ 's have the required Möbius twisting numbers on these flat strips, i.e., for all $i$ and $u \operatorname{Mtn}\left(\mathbf{a}_{u}^{i}, \mathrm{~S}_{u}^{i}\right)=$ $\operatorname{Mtn}\left(\mathbf{a}_{0}^{i}, \mathrm{~S}_{0}\right)$. Furthermore all the closed flat strips $\mathrm{S}_{u}^{i}$ are constructed such that they coincide with the planar $\varepsilon$-neighbourhoods of $\mathbf{p}_{u}$ on $\mathrm{S}_{u}$. Smoothing the edges between the closed strips $\mathrm{S}_{u}^{i}$ and the planar $\varepsilon$-disks then gives an isotopy between $\mathrm{S}_{0}$ and $\mathrm{S}_{1}$ through flat surfaces. 
Restricting $\mathrm{S}_{0}$ to a neighbourhood of the simple closed curve $\mathbf{a}_{0}^{i}$ and taking the image of this set under the given isotopy defines an isotopy between two flat closed strips, $S_{0}^{i}$ and $S_{1}^{i}$. By Lemma 8, these two flat closed strips are isotopic through flat closed strips. All except for two of the local isotopies (see the proof of Lemma 8) concern only the ruled regions of $S_{0}^{i}$ and of $S_{1}^{i}$. Hence, they do not change the planar $\varepsilon$-disks of these two surfaces. The remaining local isotopies, that ensure that neither $\mathrm{S}_{0}^{i}$ nor $\mathrm{S}_{1}^{i}$ is contained in a plane and that the self-linking numbers of their spines are equal, can be applied anywhere on these surfaces, and can therefore be kept away from their planar $\varepsilon$-disks.

The choice of rulings on the globally ruled flat strips in the isotopy between $\mathrm{S}_{0}^{i}$ and $\mathrm{S}_{1}^{i}$ constructed in the proof of Lemma 8 is (except for the segments with positive and negative torsion that can be kept away from the $\varepsilon$-disks) always orthogonal to the curves. These rulings are specified by one ruling in one point of each axis and by demanding that the corresponding ruled surfaces are flat. To each axis we now specify one ruling in a point lying in the planar $\varepsilon$-disk such that this ruling together with the tangent to the axis in this point form an ortho normal basis. of this plane. By the orthogonality of the rulings, the ruled surfaces coincide with the planar $\varepsilon$-disks. This follows from Equation 2.4, as planar curves have zero torsion.

The curves in the spines $s_{u}$ are pairwise disjoint outside the $\varepsilon$-disks. Hence, compactness ensures that a sufficiently small neighbourhood of the spines in the isotopy of flat surfaces between $\mathrm{S}_{0}$ and $\mathrm{S}_{1}$, now constructed, is in fact embedded.

By the compactness of $\mathrm{S}_{0}$, it in general consists of finitely many connected components. The proof given in the connected case carries over to the general case without changes except that one needs an index corresponding to an enumeration of the connected components. The proof of part (b) is completed.

To prove part (a) it is, as above, enough to consider the case that $\mathrm{S}$ is connected. Let $\mathrm{S}$ be a compact connected surface with nonempty boundary and let $s \subset \mathrm{S}$ be a topological spine of S. It may be assumed that $\mathrm{S}$ is planar in a neighbourhood $N$ of the point in which the curves in the spine $s$ intersect. Again, consider a spine $s$ of $\mathrm{S}$ as a finite number of closed curves $\mathbf{a}_{i}$ entering the planar $\varepsilon$-disk - each with a Möbius twisting number attached.

Claim: It may be assumed that each $\mathbf{a}_{i}$ has non-vanishing curvature. Note, that $\mathbf{a}_{i}$ has zero curvature in $\mathbf{a}_{i}(s)$ if and only if the geodesic curvature of $\mathbf{a}_{i}$ is zero in $\mathbf{a}_{i}(s)$ and $\mathbf{a}_{i}^{\prime}(s)$ is an asymptotic direction. Then the proof of this claim is analogous to the proof of Sublemma 10.

We may assume that each $\mathbf{a}_{i}$ has a $\pm \tau$-segment which is kept away from the planar part $N$ of the surface $\mathrm{S}$. This can be done preserving non-vanishing curvature.

A neighbourhood of the curve $\mathbf{a}_{i}$ on $\mathrm{S}$ is isotopic to a part of the ruled surface with $\mathbf{a}_{i}$ as axis and rulings, $\mathbf{q}_{i}$, chosen such that they together with the tangents of $\mathbf{a}_{i}$ form ortho normal bases of the tangent planes of $\mathrm{S}$ along $\mathbf{a}_{i}$. As $\mathrm{S}$ may be 
unorientable, these rulings are only projectively well-defined. Such an isotopy may be constructed using the normal exponential map and compactness.

Each $\mathbf{a}_{i}$ now fulfills the conditions of Proposition 6. Hence, a vectorfield $\mathbf{v}_{i}$ along $\mathbf{a}_{i}$ exists, such that the hereby defined surface is flat and such that the Möbius twisting number of $\mathbf{a}_{i}$ with respect to this surface is the same as the Möbius twisting number of the surface defined by $\mathbf{a}_{i}$ and $\mathbf{q}_{i}$. Furthermore, the vector field $\mathbf{v}_{i}$ may be chosen to equal $\mathbf{q}_{i}$ on the planar part $N$ of S.

Using the cylinder coordinates as in Proposition 6, the vectorfields $\mathbf{v}_{i}$ and $\mathbf{q}_{i}$ are given by $\left(\alpha_{\mathbf{v}_{i}}, \theta_{\mathbf{v}_{i}}\right)$ resp. $\left(0, \theta_{\mathbf{q}_{i}}\right)$. By construction, the surface isotopy induced by the vectorfield isotopy

$$
[0,1] \ni u \mapsto\left(u \alpha_{\mathbf{v}_{i}}, u \theta_{\mathbf{v}_{i}}+(1-u) \theta_{\mathbf{q}_{i}}\right)
$$

is the identity on the planar part on $\mathrm{S}$, and it makes a neighbourhood of the curve $\mathbf{a}_{i}$ into a flat surface. Doing this for each closed curve in the spine of S completes the proof of part (a) and hereby the proof of Theorem 13.

\section{Remark on the isotopy classes of negatively curved surfaces}

The isotopy classes of flat surfaces are described by Theorem 13 and the isotopy classes of positive curvature surfaces are described by Theorem 1 . These theorems raise the question: Is there a result analogous to Theorem 1 and Theorem 13 concerning the isotopy classes of negatively curved surfaces? The answer to this question is in the negative. As pointed out below, the lack of umbilic points on negatively curved surfaces subdivide each isotopy class of surfaces containing an orientable closed strip into countably infinitely many isotopy classes of negatively curved surfaces.

By Proposition 5, the isotopy class (with no curvature restriction) of a compact surface $\mathrm{S}$ with nonempty boundary is determined by the isotopy class of a spine $s$ of $\mathrm{S}$ with a Möbius twisting number attached to each closed curve. Assume $\mathrm{S}$ is negatively curved. By negative curvature, the principal directions corresponding to positive resp. negative principal curvature define two smooth line-fields along a closed curve on $\mathrm{S}$ in case a tubular neighbourhood of this curve on $\mathrm{S}$ is orientable.

Assume S containes an orientable closed strip and let $s$ be a spine on S. Denote by $\mathbf{a}_{i}$ a closed curve in the spine $s$ - or rather a simple closed smooth curve isotopic to $\mathbf{a}_{i}$ on $\mathrm{S}$ such that a neighbourhood of $\mathbf{a}_{i}$ on $\mathrm{S}$ is orientable. The rotation of the principal directions relative to the tangents of $\mathbf{a}_{i}$, when traversing $\mathbf{a}_{i}$ once, defines a half integer valued index. By continuity, this rotational index is independent of deformations of $\mathbf{a}_{i}$ through simple closed curves on $\mathrm{S}$ and it is independent of isotopy of $\mathrm{S}$ through negatively curved surfaces.

Figure 6 shows parts of two negatively curved ruled surfaces with the same axis. The rulings (asymptodic directions) are othogonal to this axis on both of these surfaces. There is thus no rotation of the asymptotic directions given by the rulings with respect to the axis. Hence, the rotations of the principal directions 


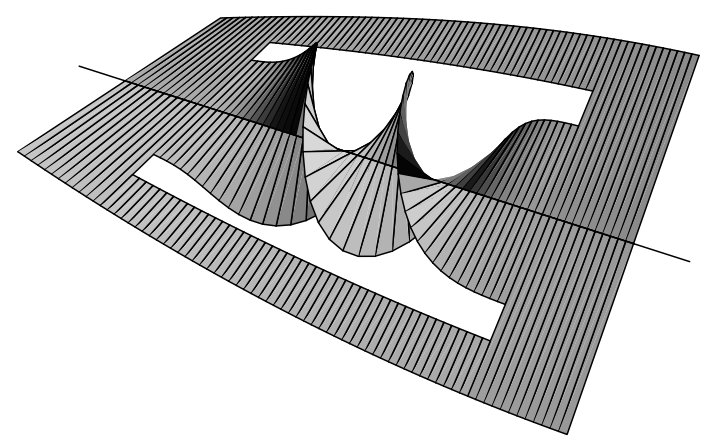

Figure 6. Parts of two negatively curved ruled surfaces with the same axis.

with respect to the axis are equal for the two surfaces. However, the contributions to the Möbius twisting number of the axis on the two surface pieces differ by one. Hence, the rotational index is independent of the Möbius twisting number. Considering isotopy of negatively curved surfaces through negatively curved surfaces, each closed curve with an orientable neighbourhood in a spine has thus an index additional to and independent of its Möbius twisting number. This causes the claimed subdivision of the isotopy classes of ordinary compact surfaces with nonempty boundary and motivates

Conjecture 14. (a) In 3-space, any compact surface with nonempty boundary is isotopic to a negatively curved surface. (b) Any two such negatively curved surfaces, $\mathrm{S}_{1}$ and $\mathrm{S}_{2}$, are isotopic through negatively curved surfaces if and only if there exists an isotopy through ordinary surfaces between $\mathrm{S}_{1}$ and $\mathrm{S}_{2}$, such that for each simple closed curve with integer Möbius twisting number on $\mathrm{S}_{1}$, this curve and its image on $\mathrm{S}_{2}$, under this isotopy, have equal rotational indices with respect to the principal directions on the respective surfaces. (These indices depend only on the regular homotopy classes of the curves.)

The reason why the rotational index does not cause subdivision of isotopy classes in the case of flat surfaces containing orientable strips or positive curvature surfaces is that non negatively curved surfaces may have umbilic points (planar regions on flat surfaces). Hence, the above rotational index is generally not welldefined on flat or positive curvature surfaces. As shown on Figure 3, the isotopies constructed in this paper use planar regions to unwind the principal directions (rulings) such that all rulings are transversal to the axes. A similar remark applies to the positive curvature model surfaces used in [3], where positive curvature strips 
are pieced together on a spherical (umbilic) surface piece. Hence, even though the flat model surfaces used here and the positive curvature model surfaces used in [3] easily can be changed into "negatively curved model surfaces", they can only produce surfaces with zero net rotation of the principal directions with respect to each closed curve with an orientable neighbourhood in a spine. All other isotopy classes of negatively curved surfaces have to be treated using other model surfaces or perhaps using entirely different methods.

\section{Acknowledgments}

I am pleased to thank the staff at U. Penn. for their hospitality during my visit. The author especially thanks Herman Gluck for posing Lemma 8 as a question and Jason Cantarella for discussions of and suggestions to proofs of Proposition 6. I would also like to thank J. Gravesen, R. Sinclair, S. Markvorsen, and V. L. Hansen for comments and sugestions to an earlier version of this manuscript.

\section{References}

[1] C. Chicone and N. J. Kalton, Flat embeddings of the Möbius strip in $\mathbb{R}^{3}$. Department of Mathematics, University of Missouri, Columbia, MO 65211, 1984.

[2] H. Gluck and L.-H. Pan, Knot theory in the presence of curvature, I. Preprint, 1994.

[3] H. Gluck and L.-H. Pan, Embedding and knotting of positive curvature surfaces in 3-space. Topology 37(4) (1998), 851-873.

[4] R. H. Kyle, Embeddings of Möbius bands in 3-dimensional space. Proc. Roy. Irish Acad. Sect. A. 57 (1955), 131-136.

[5] H. K. Moffatt and R. L. Ricca, Helicity and the Călugăreanu invariant. Proc. R. Soc. Lond. A 439 (1992), 411-429.

[6] W. Pohl, The self-linking number of a closed space curve. J. of Math. and Mechanics $\mathbf{1 7}$ (1968), 975-985.

[7] T. Randrup and P. Røgen, How to twist a knot. Arch. Math. 68 (1997), 252-264.

[8] M. Spivak, A Comprehensive Introduction to Differential Geometry, volume 3. Publish or Perish, Wilmington, second edition, 1979.

Peter Røgen

Department of Mathematics

Technical University of Denmark

Building 303

DK-2800 Lyngby

Denmark

e-mail: Peter.Roegen@mat.dtu.dk

(Received: February 29, 2000) 